\title{
The chopped moving photocarrier grating technique
}

Cite as: Rev. Sci. Instrum. 90, 123902 (2019); https://doi.org/10.1063/1.5117232

Submitted: 29 June 2019 . Accepted: 30 November 2019 . Published Online: 16 December 2019

Leonardo Kopprio (D), Federico Ventosinos (D), and Javier Schmidt (D)

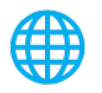

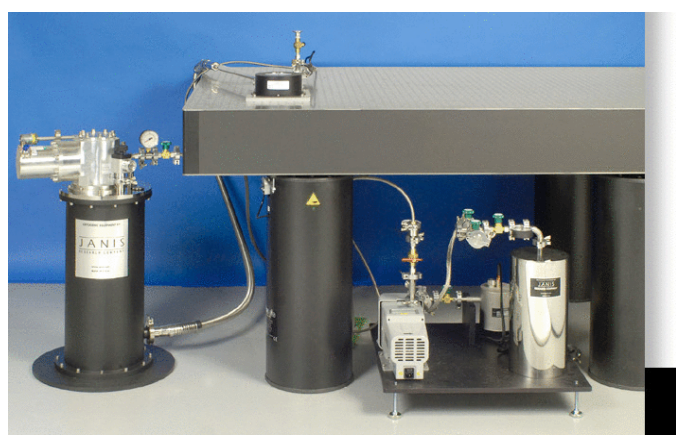

sales@janis.com

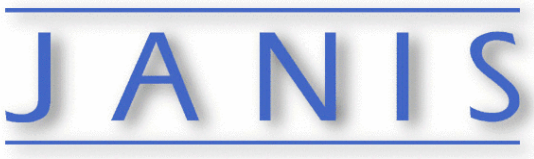

Rising LHe costs? Janis has a solution. Janis' Recirculating Cryocooler eliminates the use of Liquid Helium for "wet" cryogenic systems. 


\title{
The chopped moving photocarrier grating technique
}

\author{
Cite as: Rev. Sci. Instrum. 90, 123902 (2019); doi: 10.1063/1.5117232 \\ Submitted: 29 June 2019 • Accepted: 30 November 2019 • \\ Published Online: 16 December 2019

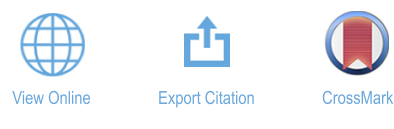

\author{
Leonardo Kopprio, ${ }^{1, a)}$ (D) Federico Ventosinos, ${ }^{1,2}$ (D) and Javier Schmidt ${ }^{1,2}$ (iD)

\begin{abstract}
AFFILIATIONS
${ }^{1}$ Instituto de Física del Litoral (IFIS-Litoral), CONICET-UNL, Güemes 3450, S3000GLN Santa Fe, Argentina
\end{abstract} \\ ${ }^{2}$ Facultad de Ingeniería Química, Universidad Nacional del Litoral, Santiago del Estero 2829, S3000AOM Santa Fe, Argentina
}

a) Electronic mail: leonardokopprio@gmail.com

\begin{abstract}
The Moving photocarrier Grating Technique (MGT) allows the simultaneous determination of the photocarrier drift mobilities and the small-signal recombination lifetime of photoconductive semiconductors. The technique measures the direct current (DC) induced by a monochromatic illumination consisting of a moving interference pattern superimposed on a uniform background of much higher intensity. A drawback of the technique is the low level of the signal to be measured, which can be masked by the noise at low temperatures or low light intensities. In this work, we propose implementing an alternating current (AC) version of the MGT by chopping the weak beam in the standard configuration. We call this new technique the Chopped Moving photocarrier Grating (CMG). In CMG, the AC signal can be measured with a lock-in amplifier for electrical noise removal. In this way, the signal-to-noise ratio can be increased compared to the standard DC technique. Assuming a multiple-trapping model for charge transport, we find the theoretical expression for the current density induced by CMG at fundamental frequency. By using a numerical simulation with parameters typical for hydrogenated amorphous silicon, we verify the expected equivalence between both techniques for low enough chopping frequencies. Then, we test experimentally this equivalence for an undoped hydrogenated amorphous silicon sample. For low signal levels, we demonstrate the superior performance of CMG.
\end{abstract}

Published under license by AIP Publishing. https://doi.org/10.1063/1.5117232

\section{INTRODUCTION}

The Moving photocarrier Grating Technique (MGT) was initially proposed by Haken et al. ${ }^{1,2}$ for determining the photocarrier drift mobilities and the common small-signal recombination lifetime $\left(\tau^{\prime}\right)$ of a photoconductive material. The technique is based on illuminating the photoconductor, in the zone between two coplanar electrical contacts, with an interference pattern (IP) moving at a constant speed from one contact to the other. A uniform illumination with a much higher intensity is superimposed to set the background conditions. The pattern movement induces a direct current (DC) on the sample (without any externally applied electric field) that depends on the speed of the movement $(v)$, the spatial period of the IP $(\Lambda)$, external parameters such as temperature and light intensity, and material parameters. The current sign is determined by the charge of the majority carrier, which makes the technique useful for the immediate determination of sample doping.

The technique was initially analyzed from a general phenomenological point of view (independent of the conduction and recombination mechanisms), without distinguishing between free and trapped charge in the material. By fitting the experimental data with the theoretical equation for the induced current as a function of $v$, the drift mobilities and the small-signal recombination lifetime were obtained. ${ }^{1,2}$ Later, Witt et al. ${ }^{3,4}$ found that when $\Lambda$ is much greater than the ambipolar diffusion length of the photocarriers and the small-signal recombination lifetime is much greater than the dielectric relaxation time,${ }^{5} \tau^{\prime}$ is simply related to the pattern speed that induces the highest current, $v_{m}$, by $\tau^{\prime}=\Lambda /\left(2 \pi v_{m}\right)$.

In the last decades, several works on the use of photoconductivity-based techniques for the characterization of semiconductors have been published. Most of these techniques were analyzed assuming that carrier transport occurs in extended states located outside the mobility-gap, whereas the localized states (within the mobility-gap) act exclusively as traps and recombination centers for the free carriers. Hopping conductivity and recombination between localized states are neglected in this multiple-trapping (MT) model. This is a good approximation for semiconductors at temperatures and generation rates not too low, and whose defect density 
is not too high, e.g., device-quality hydrogenated amorphous silicon (a-Si:H) ${ }^{6,7}$ The MT model allows relating the microscopic material parameters, like the density of states (DOS), with the photocurrent induced by the different techniques. References 8-11 include analyses of the MGT under these more specific conditions. When MGT is combined with other photoconductivity-based techniques, it can also be used for the estimation of the DOS.,11

Ventosinos et al. ${ }^{8}$ suggested that MGT is equivalent to the Oscillating Photocarrier Grating technique (OPG), i.e., the MGT current could be obtained by using another experimental configuration that produces a slightly different illumination. OPG also uses an interference pattern superimposed on a uniform background illumination of much higher intensity, although its movement is periodic: it moves at constant velocity for half a period, whereas in the next semiperiod it moves at the same speed but in the opposite direction. The oscillation of the pattern induces an alternating current (AC) in the material, without applying any external electric field. The main advantage of obtaining the MGT current using an AC measurement is related to the possibility of using a lock-in amplifier for the current measurement, which is specially designed to filter the signal from the electrical noise. Consequently, the AC measurements are particularly interesting when the signal-to-noise ratio is small. This happens especially at low temperatures or low generation rates, when the signal is small as a consequence of the small concentration of carriers in extended states.

$\mathrm{OPG},{ }^{8}$ proposed as an $\mathrm{AC}$ version of $\mathrm{MGT}$, is based on neglecting the current transients occurring after the sudden changes of the IP velocity. This is certainly a good approximation at low enough IP speeds, but may fail at high speeds (a recent discussion on OPG limitations can be found in Ref. 12). Following a similar approach, we propose here a simpler and less restrictive way of obtaining an AC measurement of the MGT current. The alternating signal is obtained by chopping the weak beam in the standard MGT configuration. We call this new technique the Chopped Moving photocarrier Grating technique (CMG), and show that it is equivalent to MGT for low enough chopping frequencies. We obtain the analytical expression for the current density at the fundamental frequency using the multiple-trapping model. The proposed equivalence between MGT and CMG for low chopping frequencies is verified for undoped a-Si:H, initially with a numerical simulation and then experimentally. Finally, we prove experimentally that CMG provides less noisy measurements for low signal conditions, performing measurements with both techniques at a low illumination level.

This paper is organized as follows. In Sec. II, we present a theoretical introduction to MGT and CMG: a brief description of the experimental setups and the basic equations is made. We also present an analysis, similar to that of Ref. 8, to find the relation between the MGT steady-state DC signal and the CMG steady-state AC signal for low enough chopping frequencies. In Sec. III, we use numerical simulations, corresponding to high quality undoped a$\mathrm{Si}: \mathrm{H}$, to compare the signals obtained from both methods, and we provide a general explanation of the obtained results. The experimental details corresponding to the measurements performed on a device-quality a-Si:H sample are given in Sec. IV. In Sec. V, we present and discuss the experimental results obtained with MGT and CMG. Section VI sums up the main conclusions of this work. Finally, the mathematical deductions are given in Appendixes A-C.

\section{THEORETICAL BACKGROUND}

Figure 1 shows a diagram of the experimental setup for MGT and CMG. A laser beam of energy higher than the bandgap of the photoconductive material is initially divided into two by a beam splitter (BS). One beam is attenuated with a neutral density filter (NDF) to produce a large difference between their intensities. The frequency of each beam is slightly modified by using acousto-optic modulators (AOM), and finally they interfere with the sample region situated between the electrical contacts. For normal incidence of one of the beams, as in Fig. $1, \Lambda$ is simply related to the angle between the interfering beams $(\delta)$ by $\Lambda=\lambda / \sin (\delta)$, where $\lambda$ is the wavelength of the laser light in air. The speed of the interference pattern is proportional to $\Lambda$ and to the frequency difference $\Delta f$ between the interfering beams, $v=\Lambda \Delta f$. The small direct current induced by MGT in the material is usually measured using an electrometer. When the weak beam is chopped (Ch), an alternating current is induced, and the configuration corresponds to CMG. The fundamental harmonic component of the AC signal is measured with a lock-in amplifier after the signal is preamplified.

When the energy of the incident photons is greater than the material gap, the mean generation rate of free-photocarriers in the material $(G)$ is proportional to the incident photon flux $(F)$ and is estimated from the following equation:

$$
G \approx \frac{(1-r)}{d}\left(1-e^{-\alpha d}\right) \times F=K \times F
$$

where $r$ is the reflection coefficient of the front surface, $d$ is the material thickness, and $\alpha$ is the material absorption coefficient for the energy of the incident photons.

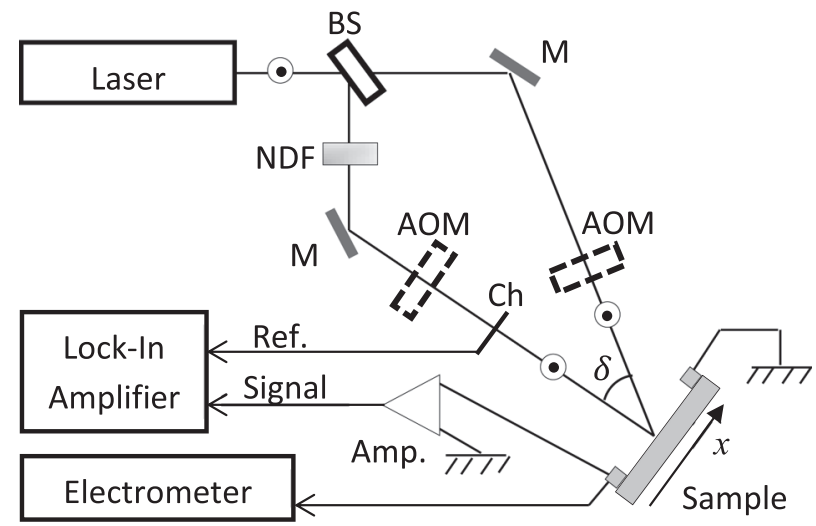

FIG. 1. Experimental setup used in MGT and CMG. A linearly polarized laser beam is divided into two by a beam splitter (BS), and then one of the beams is attenuated with a neutral density filter (NDF). The beams are made to coincide in the region between the sample contacts by using mirrors $(\mathrm{M})$. Before reaching the sample, the frequency of each beam is modified with acousto-optic modulators (AOM). The direct current induced by MGT is measured with an electrometer. If the weak beam is chopped, we get the setup for CMG. In this case, after the AC signal generated in the sample is preamplified, its fundamental harmonic component is measured with a lock-in amplifier. 


\section{A. Moving photocarrier grating technique}

The MGT generation-rate is

$$
G_{M G T}=G_{0}+\Delta G_{0} \cos (k x+\Delta \omega t)=G_{0}+\operatorname{Re}\left\{\Delta G_{0} e^{j(k x+\Delta \omega t)}\right\},
$$

where $k$ is the angular wave number, $k=2 \pi / \Lambda, \Delta \omega$ is the angular frequency difference between the interfering beams, $\Delta \omega=2 \pi \Delta f, x$ is the spatial coordinate pointing from one contact to the other, $t$ is the temporal variable, $R e$ designates the real part of a complex number, and $j$ is the imaginary unit. The generation rates in Eq. (2) are given by $G_{0}=G_{1}+G_{2}$ and $\Delta G_{0}=2 \gamma_{0} \sqrt{G_{1} G_{2}}$, where $G_{1}$ and $G_{2}$ are the mean generation rates produced by each beam separately. $\gamma_{0}$ is the interference quality factor; its value is positive and less than 1 as a consequence of light scattering, mechanical vibrations, and the partial coherence of the beams.

When there is a large difference between the photon fluxes in the beams, the condition $\Delta G_{0} \ll G_{0}$ is fulfilled, which allows discarding the higher-order terms in the equations. In the steady state, consequently, the harmonic generation rate produces free photocarrier densities with the same functional dependence on the variables, $\operatorname{Re}\left\{\Delta n_{\Delta \omega, k} e^{j(k x+\Delta \omega t)}\right\}$ and $\operatorname{Re}\left\{\Delta p_{\Delta \omega, k} e^{j(k x+\Delta \omega t)}\right\}$, and also an electric field $\operatorname{Re}\left\{\Delta \xi_{\Delta \omega, k} e^{j(k x+\Delta \omega t)}\right\}$. These functions are phase shifted from the generation rate, i.e., $\Delta n_{\Delta \omega, k}, \Delta p_{\Delta \omega, k}$, and $\Delta \xi_{\Delta \omega, k}$ are now complex numbers. In amorphous materials the photogenerated electric field is mostly produced by trapped photocarriers because they are much more abundant than free ones. The interaction of this electric field with the harmonic free photocarrier densities produces the following DC current density (without the application of an external electric field): ${ }^{8-10}$

$$
\Delta J_{M G T}=\frac{1}{2} \operatorname{Re}\left\{\Delta \sigma_{\Delta \omega, k} \Delta \xi_{\Delta \omega, k}^{*}\right\},
$$

where the photoconductivity amplitude, $\Delta \sigma_{\Delta \omega, k}$, is proportional to the amplitudes of the free photocarrier densities,

$$
\Delta \sigma_{\Delta \omega, k}=q\left(\mu_{n} \Delta n_{\Delta \omega, k}+\mu_{p} \Delta p_{\Delta \omega, k}\right) .
$$

The asterisk as a superscript defines the complex conjugate of the number, $q$ is the absolute value of the electron charge, while $\mu_{n}$ and $\mu_{p}$ are the free electron and hole mobilities. In Appendix A, we solve the transport equations within the multiple-trapping model for a slightly more general generation rate: $G=G_{0}+\operatorname{Re}\left\{g_{\Omega, \mathrm{k}} e^{j(k x+\Omega \mathrm{t})}\right\}$ with $\left|g_{\Omega, \mathrm{k}}\right| \ll G_{0}$. The expressions for $\Delta n_{\Delta \omega, k}, \Delta p_{\Delta \omega, k}$, and $\Delta \xi_{\Delta \omega, k}$ are obtained by making $g_{\Omega, k}=\Delta G_{0}$ and $\Omega=\Delta \omega$ in the equations of Appendix A.

\section{B. Chopped moving photocarrier grating technique}

The CMG generation rate is

$$
G_{C M G}=\left\{\begin{array}{ll}
G_{0}+\Delta G_{0} \cos (k x+\Delta \omega t), & t \in\left[0 ; \frac{\Gamma}{2}\right] \\
G_{1}, & t \in\left[\frac{\Gamma}{2} ; \Gamma\right]
\end{array},\right.
$$

where $G_{1}$ is produced by the more intense beam $\left(G_{1} \gg G_{2}\right)$, thus $G_{0} \approx G_{1}$. During half period the generation rate corresponds to MGT, whereas in the next semiperiod the generation rate is uniform and very close to the uniform MGT generation rate. From Maxwell's first equation it can be seen that a uniform generation rate cannot induce an electric field in the material, therefore neither can induce a current. When the chopper angular frequency, $\omega_{c}=2 \pi / \Gamma$, is sufficiently low, the current transients corresponding to the sudden variations of the generation rate after each half period can be discarded. In this case, the induced current density corresponds to a square wave, whose value goes from $\Delta J_{M G T}$ to zero as plotted in Fig. 2. When this signal reaches the lock-in amplifier, this instrument measures the fundamental frequency amplitude of the induced current density, $\Delta J_{C M G}^{\omega_{c}}$, divided by $\sqrt{2}$ (rms value). From a Fourier decomposition of the square wave, we deduce the relationship between $\left|\Delta J_{M G T}\right|$ and $\left|\Delta J_{C M G}^{\omega}\right|$,

$$
\left|\Delta J_{M G T}\right|=\frac{\pi}{2}\left|\Delta J_{C M G}^{\omega_{c}}\right|=\frac{\pi}{\sqrt{2}}\left(\frac{\left|\Delta J_{C M G}^{\omega_{c}}\right|}{\sqrt{2}}\right) .
$$

For high chopping frequencies, it is probable that the current transients distort the square wave signal presented in Fig. 2, and consequently Eq. (6) may no longer be valid.

Equation (5) can be rewritten as

$$
G=G_{1}+\left[G_{2}+\Delta G_{0} \cos (k x+\Delta \omega t)\right] f(t),
$$

where $f(t)$ is a square wave oscillating between 0 and 1 with a period $\Gamma$. Making a Fourier series expansion of $f(t)$ and using elemental trigonometric properties, we have that

$$
\begin{aligned}
G \cong & G_{1}+\frac{G_{2}}{2}+\frac{\Delta G_{0}}{2} \cos (k x+\Delta \omega t)+\frac{\Delta G_{0}}{\pi} \cos \left[k x+\left(\Delta \omega+\omega_{c}\right) t\right] \\
& +\frac{\Delta G_{0}}{\pi} \cos \left[k x+\left(\Delta \omega-\omega_{c}\right) t\right]+\frac{2}{\pi} G_{2} \cos \left(\omega_{c} t\right)
\end{aligned}
$$

where we have just kept the uniform terms and those that contribute to the induced current density at the fundamental frequency $\left(\omega_{c}\right)$. The generation rate consists of a uniform term $G_{0}^{\prime}=G_{1}$ $+G_{2} / 2 \approx G_{0}$, plus 4 harmonic functions of much smaller amplitudes. The first three harmonic functions are traveling waves of similar amplitudes; the fourth just depends on $\omega_{c} t$ and its amplitude

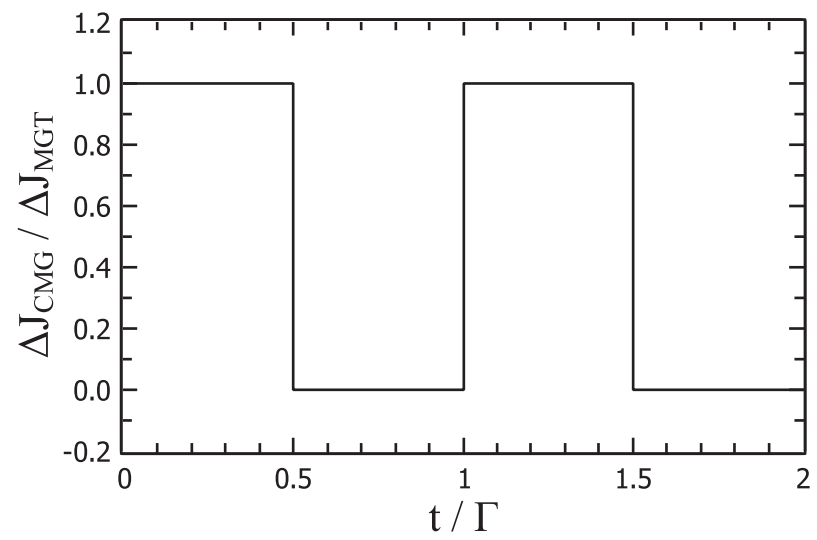

FIG. 2. Current density ratio between CMG and MGT as a function of time, if the current transients occurring after each sudden variation of the illumination are neglected. This approximation results are reasonable only for low-enough chopping frequencies. 
is even smaller than that of the others. Since $G_{0}^{\prime} \approx G_{0}$, the homogeneous photocarrier densities are approximately the same as in MGT.

The nonuniform generation rate can be rewritten using complex numbers as

$$
\Delta G=\operatorname{Re}\left\{\sum_{\Omega} g_{\Omega, k} e^{j(k x+\Omega t)}+g_{\omega_{c}, 0} e^{j \omega_{c} t}\right\},
$$

with $\Omega=\Delta \omega, \Delta \omega+\omega_{c}, \Delta \omega-\omega_{c}$, and the amplitudes of the harmonic waves being

$$
g_{\Delta \omega, k}=\frac{\Delta G_{0}}{2}, \quad g_{\Delta \omega \pm \omega_{c}, k}=\frac{\Delta G_{0}}{\pi}, \quad g_{\omega_{c}, 0}=\frac{2}{\pi} G_{2} .
$$

As a consequence of having $g_{\Delta \omega, k}, g_{\Delta \omega \pm \omega_{c}, k}, g_{\omega_{c}, 0} \ll G_{0}^{\prime}$, the nonlinear terms in the fundamental equations can be neglected. In Appendix B, we obtain the expression for the current density induced by CMG at the fundamental frequency. In the absence of an externally applied electric field, the amplitude of the fundamental frequency component is

$$
\begin{aligned}
\Delta J_{C M G}^{\omega_{c}}= & \frac{1}{2}\left[\Delta \sigma_{\Delta \omega+\omega_{c}, k} \Delta \xi_{\Delta \omega, k}^{*}+\Delta \sigma_{\Delta \omega, k}^{*} \Delta \xi_{\Delta \omega+\omega_{c}, k}\right. \\
& \left.+\Delta \sigma_{\Delta \omega, k} \Delta \xi_{\Delta \omega-\omega_{c}, k}^{*}+\Delta \sigma_{\Delta \omega-\omega_{c}, k}^{*} \Delta \xi_{\Delta \omega, k}\right] .
\end{aligned}
$$

The photoconductivity and electric field amplitudes of Eq. (11) are obtained by making $\Omega=\Delta \omega, \Delta \omega+\omega_{\mathcal{c}}$, and $\Delta \omega-\omega_{\mathrm{c}}$ successively in the equations of Appendix A, while replacing the $g_{\Omega, k}$ values with the corresponding ones given by Eq. (10).

\section{Numerical simulations}

To numerically simulate the photoconductive behavior of undoped a-Si:H, we use the following donorlike and acceptorlike densities of localized states per unit energy:

$$
\begin{aligned}
& N^{D O N}(E)=N\left(E_{V}\right) \exp \left(\frac{E_{V}-E}{k_{b} T_{V}}\right)+\frac{N_{D}}{\sqrt{2 \pi} W_{D}} \exp \left(\frac{-\left(E-E_{D}\right)^{2}}{2 W_{D}^{2}}\right), \\
& N^{A C C}(E)=N\left(E_{C}\right) \exp \left(\frac{E-E_{C}}{k_{b} T_{C}}\right)+\frac{N_{A}}{\sqrt{2 \pi} W_{A}} \exp \left(\frac{-\left(E-E_{A}\right)^{2}}{2 W_{A}^{2}}\right) .
\end{aligned}
$$

The first terms correspond to the exponential band tails originated by the dispersion of bond angles and bond lengths, as a consequence of the long-range disorder in the atomic lattice. The second terms correspond to deep Gaussian states originated in coordination defects (mostly dangling bonds). ${ }^{6,1}$

Table I presents the values of the input material parameters used in the numerical simulations, which were taken from specific literature and are widely accepted as typical for device-quality undoped a-Si:H. ${ }^{6}$ We assume that the material permittivity $\varepsilon$ is 12 times that of vacuum. For simplicity, we assume that the electron $\left(c_{n}\right)$ and hole $\left(c_{p}\right)$ capture coefficients are equal, independent of energy and the character of the state (acceptorlike or donorlike). For the density of extended states, we assume a square root dependence with energy and that they join the corresponding band tail with continuity of the function and the first derivative. In this case,
TABLE I. Values of the material parameters used in the numerical simulation, corresponding to undoped a-Si:H.

\begin{tabular}{cc}
\hline \hline Parameter & Value \\
\hline$c_{n}, c_{p}\left(\mathrm{~cm}^{3} \mathrm{~s}^{-1}\right)$ & $1 \times 10^{-8}$ \\
$\varepsilon\left(\mathrm{cm}^{-1} \mathrm{~F}\right)$ & $1.06 \times 10^{-12}$ \\
$\mu_{n}\left(\mathrm{~cm}^{2} \mathrm{~V}^{-1} \mathrm{~s}^{-1}\right)$ & 20 \\
$\mu_{p}\left(\mathrm{~cm}^{2} \mathrm{~V}^{-1} \mathrm{~s}^{-1}\right)$ & 2 \\
$N\left(E_{V}\right), N_{C}\left(E_{C}\left(\mathrm{~cm}^{-3} \mathrm{eV}^{-1}\right)\right.$ & $2 \times 10^{21}$ \\
$N_{A}, N_{D}\left(\mathrm{~cm}^{-3}\right)$ & $2 \times 10^{16}$ \\
$W_{A}, W_{D}(\mathrm{eV})$ & 0.3 \\
$E_{D}(\mathrm{eV})$ & 0.9 \\
$E_{A}(\mathrm{eV})$ & 1.15 \\
$E_{C}-E_{V}(\mathrm{eV})$ & 1.8 \\
$T_{C}(\mathrm{~K})$ & 280 \\
$T_{V}(\mathrm{~K})$ & 550 \\
\hline \hline
\end{tabular}

the effective densities of extended states in the conduction $(C)$ band edge is given by

$$
\begin{aligned}
N_{C}= & N\left(E_{C}\right) k_{b} T \sqrt{\frac{\pi T}{2 T_{C}}} \exp \left(\frac{T_{C}}{2 T}\right) \\
& \times\left(1-\operatorname{erf}\left(\sqrt{\frac{T_{C}}{2 T}}\right)+\sqrt{\frac{2 T_{C}}{\pi T}} \exp \left(\frac{-T_{C}}{2 T}\right)\right),
\end{aligned}
$$

where $\operatorname{erf}()$ is the error function. ${ }^{14}$ The equation for the effective density of extended states in the valence band edge is similar and can be obtained by replacing the subindex $C$ with $V$ in the above equation.

The numerical simulation starts by solving simultaneously the charge neutrality and continuity equations given by (A15) and (A16), respectively, to obtain the uniform free carrier concentrations, $n_{0}$ and $p_{0}$, for certain temperature and uniform generation rate. Then, we obtain $\Delta n_{\Delta \omega, k}$ and $\Delta p_{\Delta \omega, k}$ making $g_{\Omega, k}=\Delta G_{0}$ and $\Omega=\Delta \omega$ in Eqs. (A25) and (A26). Replacing Eqs. (A18) and (4) with Eq. (3), we find the MGT current density. To find $\Delta J_{C M G}^{\omega_{c}}$, we need to obtain $\Delta n_{\Omega, k}$ and $\Delta p_{\Omega, k}$ with the corresponding $g_{\Omega, k}$ value given by Eq. (10), for $\Omega=\Delta \omega, \Delta \omega+\omega_{\mathrm{c}}$, and $\Delta \omega-\omega_{c}$. Replacing the values obtained for $\Delta \sigma_{\Omega, k}$ and $\Delta \xi_{\Omega, k}$ in Eq. (11), we get the fundamental frequency component of the induced CMG current density. Finally, Eq. (6) is used to link CMG to MGT.

The results plotted in Figs. 3 and 4 were obtained for $T=200 \mathrm{~K}$, $G_{0}=5 \times 10^{20} \mathrm{~cm}^{-3} \mathrm{~s}^{-1}$, and $\Lambda=6 \times 10^{-4} \mathrm{~cm}$, although the general tendencies mentioned below are independent of these precise values. These figures present the MGT steady-state current density vs the angular frequency difference between interfering beams. The solid lines were obtained with Eq. (3), whereas the dashed lines were computed with Eq. (6) and the CMG current density calculated from Eq. (11). Figure 3 confirms the validity of Eq. (6), and therefore the equivalence between CMG and MGT for low enough chopping frequencies. Figure 4 presents results obtained for higher chopping frequencies. To simplify the examination of the figure, we highlight with a vertical line the frequency difference, $\Delta \omega$, which matches the chopping frequency $\left(\omega_{c}\right)$ used for the obtainment of each dashed 


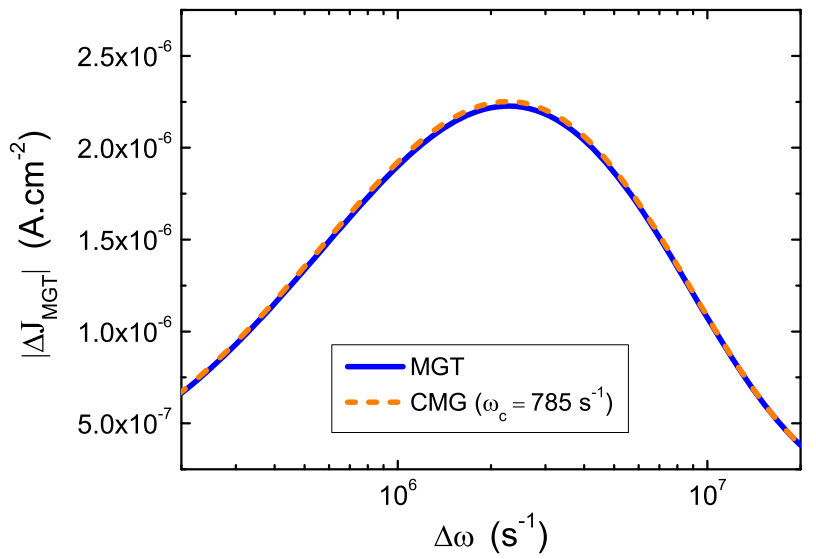

FIG. 3. MGT steady-state current density vs the frequency difference between interfering beams obtained from the numerical simulation for undoped a-Si:H. The solid line corresponds to the exact value obtained with $\mathrm{Eq}$. (3), whereas the dashed line was computed with Eq. (6), and the CMG current density was calculated from Eq. (11) with a low chopping angular frequency of $785 \mathrm{~s}^{-1}$.

line. We can see that, for $\Delta \omega \leq \omega_{c}$, the value of $\left|\Delta J_{M G T}\right|$ obtained with Eq. (6) moves away from the exact value, although for $\Delta \omega$ $\gg \omega_{c}$, we observe again the superposition of both curves.

In Appendix C, we present a formal general proof of Eq. (6) for chopping frequencies much smaller than the frequency difference between interfering beams $\left(\omega_{c} \ll \Delta \omega\right)$, where we can appreciate that the equivalence between both techniques is independent of the precise values of the material parameters. Figure 5 presents a qualitative picture of the signal induced by CMG in a photoconductive material more general than Fig. 2, where we can also visualize how the signal gets distorted when the chopping frequency is increased.

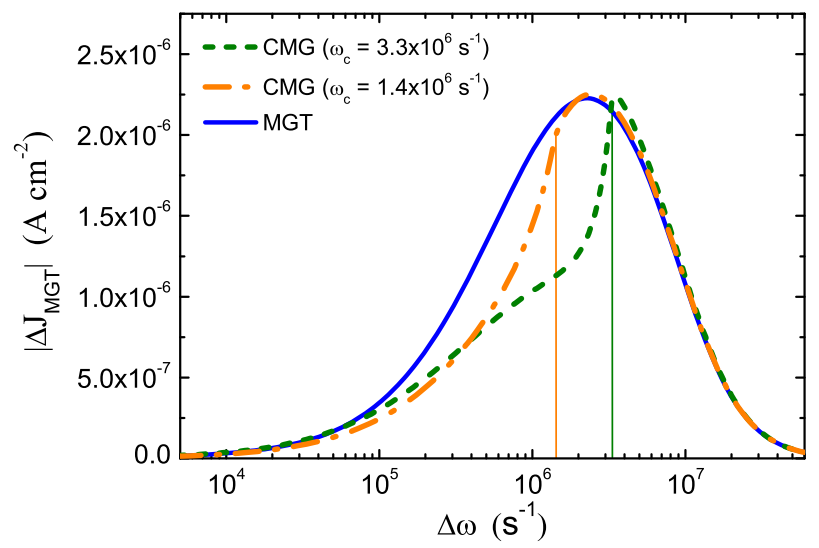

FIG. 4. MGT steady-state current density vs the frequency difference between interfering beams obtained with a numerical simulation for undoped a-Si:H. The solid line corresponds to the exact value obtained with Eq. (3), whereas the dashed lines were obtained with Eq. (6) and the CMG current density calculated from Eq. (11) using the high chopping frequencies indicated in the inset. The vertical lines correspond to the $\Delta \omega$ values that match the chopping frequency $\left(\omega_{c}\right)$ used for the obtainment of each dashed line.

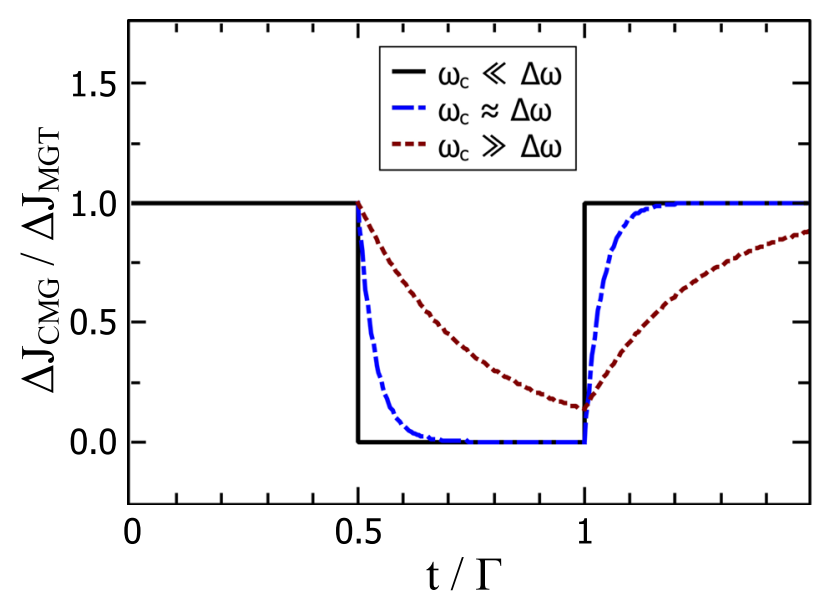

FIG. 5. Qualitative plot of the signals induced by CMG for different chopping frequencies. The interference pattern is initially moving at constant speed, so that the current density already achieved its steady-state value at $t=0$. At $t=\Gamma / 2$, the interference pattern is destroyed by blocking the weak beam. At $t=\Gamma$, the weak beam is unblocked and the interference pattern is again reconstructed.

In Fig. 5, the interference pattern is initially moving at constant velocity, and the induced current has already reached its steadystate value at $t=0$. As in CMG, the weak beam is blocked at $t=\Gamma / 2$ and is again unblocked at $t=\Gamma$. Due to the inertia and recombination lifetime of the photocarriers, a transient is produced after the destruction or reconstruction of the IP, which induces the current. For simplicity we assume the same exponential transient after the destruction and the reconstruction of the IP. For low enough chopping frequencies, the duration of the transient $\left(\tau_{t}\right)$ is negligible compared to the time that the generation rate remains the same $(\Gamma / 2$ $\left.=\pi / \omega_{c}\right)$ and the signal induced by CMG is square $\left(\tau_{t} \ll \Gamma / 2\right)$. Rising the chopper frequency (which is the same as decreasing the period $\Gamma$ ) produces a reduction of the time scale in Fig. 5. After increasing the frequency, $\omega_{c}$, a certain amount, the transients begin to be noticed in the figure and the square signal is slightly distorted. However, the steady-state current value, $\Delta J_{M G T}$, is reached before the sudden change of the generation rate $\left(\tau_{t}<\Gamma / 2\right)$. If we keep increasing the chopping frequency, after a certain point the steady-state value is no longer reaches in a half period $\left(\tau_{t}>\Gamma / 2\right)$, and the shape of the signal is entirely defined by the transients. The three lines plotted in Fig. 5 correspond to the three regimes described. From the numerical simulations (see Fig. 4), it can be observed that Eq. (6) is strictly valid for $\omega_{c} \ll \Delta \omega$, approximately valid for $\omega_{c} \approx \Delta \omega$, and invalid for $\omega_{c} \gg \Delta \omega$. Consequently, we can associate the three regimes plotted in Fig. 5 with each frequency range. It can be shown by a Fourier decomposition of the higher chopping frequency signals of Fig. 5 that the exponential transients produce a reduction of $\left|\Delta J_{C M G}^{\omega_{c}}\right|$, which is consistent with the underestimation of the exact $\left|\Delta J_{M G T}\right|$ value observed in Fig. 4 for $\omega_{c}>\Delta \omega$. $^{1 .}$

\section{EXPERIMENTAL METHODS}

To test experimentally the equivalence between CMG for low chopping frequencies and MGT, we made some measurements on an undoped a-Si:H sample. The material was deposited on a 
Corning 1737 glass substrate by PECVD in a conventional capacitively coupled reactor operated at $50 \mathrm{MHz}$, from pure silane at a flux of 40 SCCM, a chamber pressure of 0.53 Torr and a substrate temperature of $250^{\circ} \mathrm{C}$. Two coplanar silver electrodes, with a height of $1 \mathrm{~cm}$ and a separation between them of $1 \mathrm{~mm}$, were painted on the material. After a thermal annealing at $150{ }^{\circ} \mathrm{C}$, the ohmicity of the electrical contacts was checked. Finally, the material was light soaked homogeneously for $2 \mathrm{~h}$ at $T \sim 160 \mathrm{~K}$ with a $100 \mathrm{~W}$ tungsten lamp to minimize the changes of the material transport parameters induced by light during the measurements.

A linearly polarized He-Ne laser $(633 \mathrm{~nm} / 1.96 \mathrm{eV})$ was used as light source, because its energy is greater than the mobility-gap energy of device-quality a-Si: $\mathrm{H}(1.8 \mathrm{eV})$. The acousto-optic modulators (IntraAction AOM404A1) produce a slight angular deviation of the laser light, proportional to the frequency shift they induce on the light beam. For this reason, the frequency variations between interfering beams, $\Delta f$, were obtained by modifying the frequency of just one beam, whose modulator was placed nearby the sample to avoid that the angular deviations affect the beam position between the electrodes. The photon flux was measured with a calibrated silicon photodiode, whereas the other parameters needed for the generation rate estimation were obtained from the interference pattern of the UV-vis $(400-1750 \mathrm{~nm})$ transmittance and reflectance spectra [see Eq. (1)]. We obtained $r=0.48, d=0.49 \mu \mathrm{m}$ and $\alpha=1.7 \times 10^{4} \mathrm{~cm}^{-1}$.

MGT and CMG measurements were performed at room temperature with grating periods $\Lambda$ between 0.75 and $2.7 \mu \mathrm{m}$, and a ratio between beam photon fluxes greater than 20. For MGT we used a system electrometer (Keithley 6514) to measure the DC current. For CMG we used a current preamplifier (Femto DLPCA-200) and a lock-in amplifier (Stanford Research Systems SR830) to measure the AC current. The chopping frequency for the weak beam was $125 \mathrm{~Hz}$ (the same used in Fig. 3) in some experiments and $77 \mathrm{~Hz}$ in others.

\section{EXPERIMENTAL RESULTS AND DISCUSSION}

Figure 6 presents the normalized current densities obtained for two different uniform photon fluxes, $10^{16}$ and $2 \times 10^{16} \mathrm{~cm}^{-2} \mathrm{~s}^{-1}$, which produce a mean uniform generation rate in the material of $6 \times 10^{19}$ and $1.2 \times 10^{20} \mathrm{~cm}^{-3} \mathrm{~s}^{-1}$, respectively. The empty triangles were obtained by directly measuring the MGT current (DC), whereas the full triangles were found using Eq. (6) with the measurement of the current induced by $\mathrm{CMG}$ at the fundamental frequency (AC). Notice that the positions of the maxima, $\Delta \omega_{m}$, used for the estimation of the small-signal recombination lifetime $\left(\tau^{\prime} \approx \Delta \omega_{m}^{-1}\right), 3,4$ are the same in both techniques. Moreover, the shapes of the curves, used for the estimation of $\tau^{\prime}$ and the drift mobilities, ${ }^{1,2}$ are also very similar. As expected, the largest differences appear at small current values, where experimental errors become more important.

To perform a comparison between MGT and CMG under more demanding conditions, we performed measurements at room temperature using a much lower photon flux of $6.6 \times 10^{14} \mathrm{~cm}^{-2} \mathrm{~s}^{-1}$. In this case, the signals are of the order of a tenth of a picoampere due to the much lower carrier densities. For such low signals, it is expected that the AC technique would provide a better signal-tonoise ratio. The experiment was performed measuring ten curves with each technique, and then calculating the mean value and the

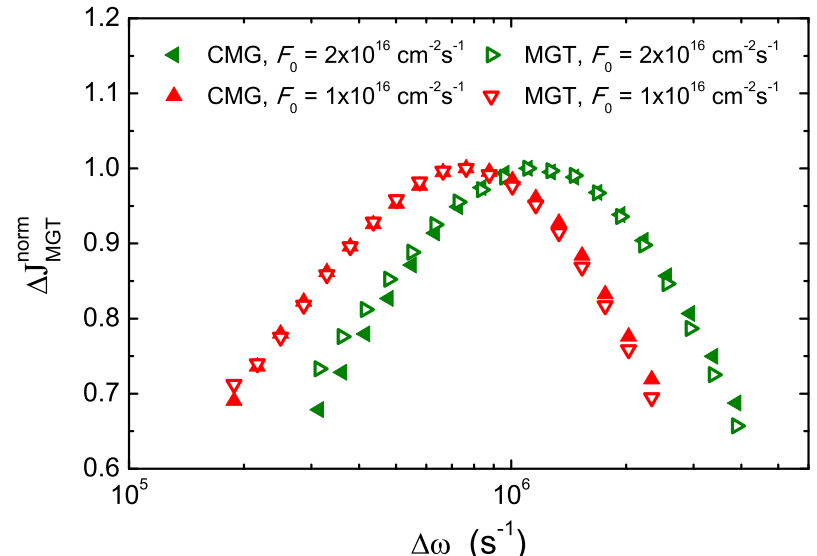

FIG. 6. Experimental curves of normalized induced current as a function of the angular frequency difference between the interfering beams, obtained at ambient temperature for two different uniform photon fluxes and a grating period $\Lambda=2.7 \mu \mathrm{m}$. The empty triangles were acquired directly from MGT, whereas the filled ones were obtained with Eq. (6) and the CMG current measured at the fundamental frequency with a chopper frequency of $125 \mathrm{~Hz}$.

standard deviation for each point. The results are presented in Fig. 7, where the mean current flowing between the contacts is plotted as a function of the angular frequency difference between the interfering beams. The error bars correspond to the standard deviation for each point, which in the case of CMG are smaller than the size of the symbols. As can be seen, the CMG curve is well defined and the error bars are much lower than for the MGT. Also, the position of the peak is much better defined using the CMG technique.

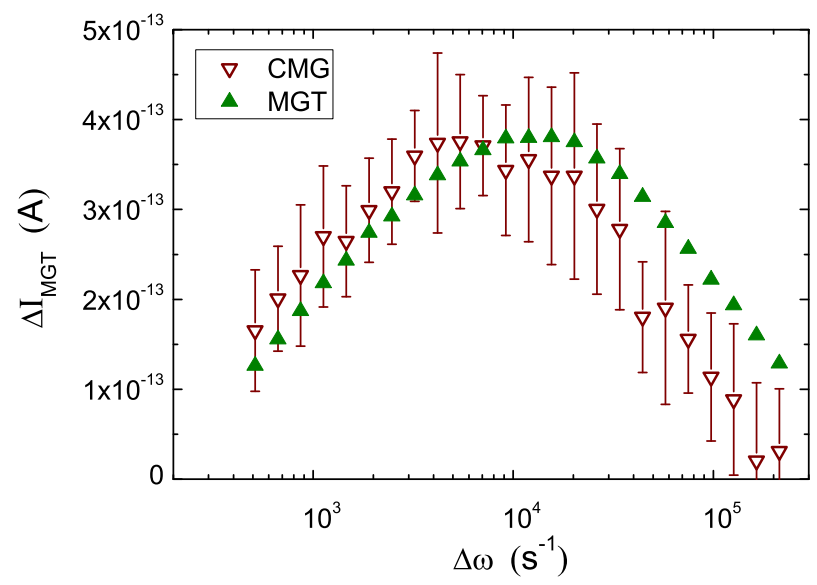

FIG. 7. Experimental curves of induced current as a function of the angular frequency difference between the interfering beams, obtained at ambient temperature for a flux of $6.6 \times 10^{14} \mathrm{~cm}^{-2} \mathrm{~s}^{-1}$ and a grating period $\Lambda=0.75 \mu \mathrm{m}$. The empty triangles correspond to the MGT current measured with an electrometer, whereas the solid triangles were obtained with Eq. (6) and the CMG current measured with a lock-in amplifier at the fundamental frequency of $77 \mathrm{~Hz}$. The error bars correspond to the standard deviation of ten measurements, which for $\mathrm{CMG}$ are smaller than the size of the points. 
Therefore, it is clear that the CMG technique performs better under low signal conditions. The same behavior would be expected at low temperatures, where the signal also decreases due to the low carrier concentrations. ${ }^{10}$

In this work, we showed that for chopping frequencies much smaller than the frequency difference between interfering beams $\left(\omega_{c} \ll \Delta \omega\right)$, the steady-state MGT current can be obtained with Eq. (6) by measuring the AC current induced by CMG at the fundamental frequency. Although we proved it, experimentally and through numerical simulations, for high-quality undoped a-Si:H, these results should be valid independent of the particular characteristics of the photoconductive material. Notice that this equation was obtained from Fig. 2, which is independent of the specific characteristics of the photoconductor.

\section{CONCLUSIONS}

In this work, we propose the chopped moving photocarrier grating (CMG) technique as an AC implementation of the moving photocarrier grating technique (MGT). The main advantage of measuring an AC signal instead of a direct current is related to the possibility of using a lock-in amplifier for the electrical noise removal, obtaining in this way a better signal-to-noise ratio. As a consequence, the proposed technique is especially useful for low temperatures or low generation rates, where the induced MGT direct current is usually too small to be directly measured with precision.

The CMG alternating signal is obtained by chopping the weak beam in the standard MGT configuration at a low enough frequency. Initially, we deduce the expression of the current density induced by $\mathrm{CMG}$ at the fundamental frequency by using the multiple-trapping model. Then, we find through numerical simulation (for undoped a-Si:H) that both techniques are equivalent only when the chopping frequency is much lower than the frequency difference between the beams $\left(\omega_{c} \ll \Delta \omega\right)$. Finally, MGT and CMG for $\omega_{c} \ll \Delta \omega$ are implemented on an undoped a-Si:H sample under the same experimental conditions. When the signal is large, the shape of the curves and the position of the maximum are the same in both techniques, thus confirming the theoretical expectations. Under low-signal conditions, a much better signal-to-noise ratio of CMG is clearly manifested.

\section{ACKNOWLEDGMENTS}

We thank R. Koropecki for valuable discussions. We acknowledge support from the Argentinian National Scientific and Technical Research Council under Project Nos. PUE 22920160100054CO and PIP 0414; Argentinian National Agency for Science and Technology Promotion under Project No. PICT-2016-1389; and National University of the Littoral under Project No. CAI+D 50420150100108LI.

\section{APPENDIX A: HARMONIC TRAVELING WAVE ON A MUCH GREATER UNIFORM BACKGROUND}

Let us suppose an arbitrary monovalent density of localized states in the gap, consisting of a donor plus an acceptor distribution,

$$
N(E)=N^{D O N}(E)+N^{A C C}(E) .
$$

For simplicity, we assume constant capture coefficients independent of the state energy and character. The free carrier electron $(n)$ and hole $(p)$ densities for certain absolute temperature $(T)$ and freecarrier generation rate $(G)$ are obtained by solving the system of equations composed of the continuity equation for each free carrier and the Maxwell's first equation,

$$
\begin{gathered}
\frac{\partial n}{\partial t}=G-r_{n}+\mu_{n}\left(\frac{\partial n}{\partial x} \xi+n \frac{\partial \xi}{\partial x}\right)+\frac{k_{b} T}{q} \mu_{n} \frac{\partial^{2} n}{\partial x^{2}}, \\
\frac{\partial p}{\partial t}=G-r_{p}-\mu_{p}\left(\frac{\partial p}{\partial x} \xi+p \frac{\partial \xi}{\partial x}\right)+\frac{k_{b} T}{q} \mu_{p} \frac{\partial^{2} p}{\partial x^{2}}, \\
\frac{\partial \xi}{\partial x}=\frac{q}{\varepsilon}\left(p+p^{t}-n-n^{t}\right),
\end{gathered}
$$

where $r_{n}$ is the recombination rate of the free electrons,

$$
r_{n}=\int_{E_{V}}^{E_{C}}\left[f^{p}(E) c_{n} n-f^{n}(E) e_{n}(E)\right] N(E) d E
$$

and $r_{p}$ is the recombination rate of free holes, whose definition can be obtained replacing $\mathrm{p}$ by $\mathrm{n}$ and vice versa in Eq. (A5). $p^{t}$ and $n^{t}$ are the positive and negative densities of localized states, respectively,

$$
p^{t}=\int_{E_{V}}^{E_{C}} f^{p}(E) N_{D O N}(E) d E, \quad n^{t}=\int_{E_{V}}^{E_{C}} f^{n}(E) N_{A C C}(E) d E .
$$

$f^{n}(E)$ and $f^{p}(E)$ are the fraction of states occupied with electrons and holes, respectively; therefore,

$$
f^{n}(E)+f^{p}(E)=1 .
$$

Within the multiple-trapping model the electron occupation function is given by

$$
\frac{\partial f^{n}(E)}{\partial t}=c_{n} n+e_{p}(E)-f^{n}(E)\left(c_{n} n+c_{p} p+e_{n}(E)+e_{p}(E)\right),
$$

where $e_{n}(E)$ and $e_{p}(E)$ are the emission coefficients, which are directly related to capture coefficients as a consequence of the detailed balance principle, ${ }^{16,17}$

$$
\begin{aligned}
& e_{n}(E)=c_{n} N_{C} \exp \left(\frac{E-E_{C}}{k_{b} T}\right), \\
& e_{p}(E)=c_{p} N_{V} \exp \left(\frac{E_{V}-E}{k_{b} T}\right) .
\end{aligned}
$$

Suppose a generation rate consisting of a sinusoidal traveling wave superimposed on a much greater uniform background $\left(g_{\Omega, k} \ll G_{0}\right)$,

$$
G=G_{0}+\operatorname{Re}\left\{g_{\Omega, k} e^{j(k x+\Omega t)}\right\} .
$$

Considering that $g_{\Omega, k}$ is a small perturbation to $G_{0}$, the same functional dependence is expected for the free carrier densities, the occupation functions, and the electric field,

$$
n=n_{0}+\operatorname{Re}\left\{\Delta n_{\Omega, k} e^{j(k x+\Omega t)}\right\}, \quad p=p_{0}+\operatorname{Re}\left\{\Delta p_{\Omega, k} e^{j(k x+\Omega t)}\right\},
$$




$$
\begin{gathered}
f^{n}=f_{0}^{n}+\operatorname{Re}\left\{\Delta f_{\Omega, k}^{n} e^{j(k x+\Omega t)}\right\}, \quad f^{p}=f_{0}^{p}+\operatorname{Re}\left\{\Delta f_{\Omega, k}^{p} e^{j(k x+\Omega t)}\right\}, \\
\xi=\xi_{\text {ext }}+\operatorname{Re}\left\{\Delta \xi_{\Omega, k} e^{j(k x+\Omega t)}\right\} .
\end{gathered}
$$

Replacing Eqs. (A11)-(A14) in the previous equations and equating the uniform and harmonics terms separately, we obtain

$$
\begin{gathered}
p_{0}+\int_{E_{V}}^{E_{C}} f_{0}^{p}(E) N^{D O N}(E) d E-n_{0}-\int_{E_{V}}^{E_{C}} f_{0}^{n}(E) N^{A C C}(E) d E=0, \\
G_{0}-\left(n_{0} p_{0}-N_{C} N_{V} e^{\frac{E_{V}-E_{C}}{k_{b} T}}\right) \int_{E_{V}}^{E_{C}} c_{n} c_{p} \tau_{0}(E) N(E) d E=0, \\
W_{\Omega, k}^{1} \Delta n_{\Omega, k}+W_{\Omega, k}^{2} \Delta p_{\Omega, k}=g_{\Omega, k}, \quad W_{\Omega, k}^{3} \Delta n_{\Omega, k}+W_{\Omega, k}^{4} \Delta p_{\Omega, k}=g_{\Omega, k}, \\
\Delta \xi_{\Omega, k}=\frac{j q}{k \varepsilon}\left[\left(1+Q_{\Omega}^{-}\right) \Delta n_{\Omega, k}-\left(1+Q_{\Omega}^{+}\right) \Delta p_{\Omega, k}\right],
\end{gathered}
$$

where the uniform occupation functions are

$$
f_{0}^{n}(E)=1-f_{0}^{p}(E)=\left(c_{n} n_{0}+e_{p}(E)\right) \tau_{0}(E),
$$

with $\tau_{0}(E)=\left[c_{n} n_{0}+c_{p} p_{0}+e_{n}(E)+e_{p}(E)\right]^{-1}$. The parameters $Q_{\Omega}^{-}$and $Q_{\Omega}^{+}$are related to the total trapped charge and are given by

$$
Q_{\Omega}^{-}=\int_{E_{v}}^{E_{c}} \frac{c_{n} f_{0}^{p}(E) N(E)}{1 / \tau_{0}(E)+j \Omega} d E, \quad Q_{\Omega}^{+}=\int_{E_{v}}^{E_{c}} \frac{c_{p} f_{0}^{n}(E) N(E)}{1 / \tau_{0}(E)+j \Omega} d E .
$$

The frequencies in Eq. (A17) are given by

$$
\begin{gathered}
W_{\Omega, k}^{1}=j\left(\Omega-k \mu_{n} \xi_{\text {ext }}\right)+k^{2} \frac{k_{b} T}{q} \mu_{n}+\frac{\left(1+Q_{\Omega}^{-}\right)}{\varepsilon} q \mu_{n} n_{0} \\
+\int_{E_{v}}^{E_{c}} c_{n}\left(\frac{c_{p} p_{0}+e_{p}(E)+j \Omega}{1 / \tau_{0}(E)+j \Omega}\right) f_{0}^{p}(E) N(E) d E, \quad(\mathrm{~A} 21) \\
W_{\Omega, k}^{2}=-\frac{\left(1+Q_{\Omega}^{+}\right)}{\varepsilon} q \mu_{n} n_{0}+\int_{E_{v}}^{E_{c}} c_{p}\left(\frac{c_{n} n_{0}+e_{n}(E)}{1 / \tau_{0}(E)+j \Omega}\right) f_{0}^{n}(E) N(E) d E, \\
W_{\Omega, k}^{3}=-\frac{\left(1+Q_{\Omega}^{-}\right)}{\varepsilon} q \mu_{p} p_{0}+\int_{E_{v}}^{E_{c}} c_{n}\left(\frac{c_{p} p_{0}+e_{p}(E)}{1 / \tau_{0}(E)+j \Omega}\right) f_{0}^{p}(E) N(E) d E, \\
W_{\Omega, k}^{4}=j\left(\Omega+k \mu_{p} \xi_{e x t}\right)+k^{2} \frac{k_{b} T}{q} \mu_{p}+\frac{\left(1+Q_{\Omega}^{+}\right)}{\varepsilon} q \mu_{p} p_{0} \\
+\int_{E_{v}}^{E_{c}} c_{p}\left(\frac{c_{n} n_{0}+e_{n}(E)+j \Omega}{1 / \tau_{0}(E)+j \Omega}\right) f_{0}^{n}(E) N(E) d E . \quad(\mathrm{A} 24)
\end{gathered}
$$

At a given absolute temperature $T$, the steady-state free electron $\left(n_{0}\right)$ and hole $\left(p_{0}\right)$ densities, corresponding to the uniform part of the generation rate $G_{0}$, are determined by the charge neutrality and the continuity equations, given by Eqs. (A15) and (A16), respectively.
The harmonic amplitudes of the free-carrier densities are obtained by solving the linear system of Eq. (A17),

$$
\begin{gathered}
\Delta n_{\Omega, k}=\frac{g_{\Omega, k}\left(W_{\Omega, k}^{4}-W_{\Omega, k}^{2}\right)}{W_{\Omega, k}^{1} W_{\Omega, k}^{4}-W_{\Omega, k}^{2} W_{\Omega, k}^{3}}, \\
\Delta p_{\Omega, k}=\frac{g_{\Omega, k}\left(W_{\Omega, k}^{1}-W_{\Omega, k}^{3}\right)}{W_{\Omega, k}^{1} W_{\Omega, k}^{4}-W_{\Omega, k}^{2} W_{\Omega, k}^{3}} .
\end{gathered}
$$

The harmonic amplitude of the electric field is obtained by replacing $\Delta n_{\Omega, k}$ and $\Delta p_{\Omega, k}$ in Eq. (A18), whereas the harmonic amplitude of the photoconductivity is given by

$$
\Delta \sigma_{\Omega, k}=q\left(\mu_{n} \Delta n_{\Omega, k}+\mu_{p} \Delta p_{\Omega, k}\right) .
$$

When the nonuniform generation rate depends only on time, $k=0$ in Eq. (A11), the harmonic amplitudes of the free-carrier densities are also given by Eqs. (A25) and (A26), but the frequencies are simpler,

$$
\begin{gathered}
W_{\Omega, 0}^{1}=j \Omega+\int_{E_{v}}^{E_{c}} c_{n}\left(\frac{c_{p} p_{0}+e_{p}(E)+j \Omega}{1 / \tau_{0}(E)+j \Omega}\right) f_{0}^{p}(E) N(E) d E, \\
W_{\Omega, 0}^{2}=\int_{E_{v}}^{E_{c}} c_{p}\left(\frac{c_{n} n_{0}+e_{n}(E)}{1 / \tau_{0}(E)+j \Omega}\right) f_{0}^{n}(E) N(E) d E, \\
W_{\Omega, 0}^{3}=\int_{E_{v}}^{E_{c}} c_{n}\left(\frac{c_{p} p_{0}+e_{p}(E)}{1 / \tau_{0}(E)+j \Omega}\right) f_{0}^{p}(E) N(E) d E,
\end{gathered}
$$

and

$$
W_{\Omega, 0}^{4}=j \Omega+\int_{E_{v}}^{E_{c}} c_{p}\left(\frac{c_{n} n_{0}+e_{n}(E)+j \Omega}{1 / \tau_{0}(E)+j \Omega}\right) f_{0}^{n}(E) N(E) d E .
$$

From Maxwell's first equation, we can see that there is no induced electric field in this case $\left(\Delta \xi_{\Omega, 0}=0\right)$.

\section{APPENDIX B: CURRENT DENSITY INDUCED BY CMG AT THE FUNDAMENTAL FREQUENCY}

As a consequence of having $g_{\Delta \omega, k}, g_{\Delta \omega \pm \omega_{c}, k}, g_{\omega_{c}, 0} \ll G_{0}^{\prime}$, the nonlinear terms in the fundamental equations can be neglected. Then, the photoconductivity and the induced electric field can be found by adding the solutions obtained for each term of Eq. (9),

$$
\begin{gathered}
\Delta \sigma=\operatorname{Re}\left\{\sum_{\Omega} \Delta \sigma_{\Omega, k} e^{j(k x+\Omega t)}+\Delta \sigma_{\omega_{c}, 0} e^{j \omega_{c} t}\right\}, \\
\Delta \xi=\sum_{\Omega} \operatorname{Re}\left\{\Delta \xi_{\Omega, k} e^{j(k x+\Omega t)}\right\} .
\end{gathered}
$$

The expressions for $\Delta \sigma_{\Omega, k}$ and $\Delta \xi_{\Omega, k}$ are obtained in Appendix A. Note that the fourth term of Eq. (9) does not induce an electric field, as was explained in the last paragraph of Appendix A. 
As a consequence of the spatial periodicity, the mean total current density coincides with the average current density over one period,

$$
\begin{aligned}
J & =\frac{1}{\mathrm{~h}} \int_{0}^{h}\left[q\left(\mu_{n} n+\mu_{p} p\right) \xi+k_{b} T\left(\mu_{n} \frac{\partial n}{\partial x}-\mu_{p} \frac{\partial p}{\partial x}\right)\right] d x \\
& =\frac{1}{\Lambda} \int_{0}^{\Lambda} q\left(\mu_{n} n+\mu_{p} p\right) \xi d x=J_{0}+\Delta J_{C M G},
\end{aligned}
$$

where $h$ corresponds to the distance between electrical contacts. Note that the diffusion currents (second term inside the square bracket) does not contribute to the mean current. $J_{0}$ is the current density produced by the uniform generation rate $G_{0}^{\prime}$, and is null when the externally applied electric field $\xi_{\text {ext }}$ is zero,

$$
J_{0}=\frac{1}{\Lambda} \int_{0}^{\Lambda} q\left(\mu_{n} n_{0}+\mu_{p} p_{0}\right) \xi_{\text {ext }} d x=\sigma_{0} \xi_{\text {ext }}
$$

$\Delta J_{C M G}$ corresponds to the current density induced by the nonuniform part of the generation rate,

$$
\begin{aligned}
\Delta J_{C M G}= & R e\left\{\Delta \sigma_{\omega_{c}, 0} e^{j \omega_{c} \mathrm{t}}\right\}_{e x t}+\frac{1}{\Lambda} \int_{0}^{\Lambda} \sum_{\Omega} \operatorname{Re}\left\{\Delta \sigma_{\Omega, k} e^{j(k x+\Omega t)}\right\} \\
& \times \sum_{\Omega^{\prime}} \operatorname{Re}\left\{\Delta \xi_{\Omega^{\prime}, k} e^{j\left(k x+\Omega^{\prime} t\right)}\right\} d x
\end{aligned}
$$

which can be rewritten as

$$
\Delta J_{C M G}=\Delta J_{C M G}^{0}+\operatorname{Re}\left\{\Delta J_{C M G}^{\omega_{c}} e^{j \omega_{c} \mathrm{t}}\right\}+\operatorname{Re}\left\{\Delta J_{C M G}^{2 \omega_{c}} e^{j 2 \omega_{c} \mathrm{t}}\right\}+\cdots
$$

Using the property $\operatorname{Re}\{C\}=\left(C+C^{*}\right) / 2$, for an arbitrary complex number $C$ and its complex conjugate $C^{*}$, the current density component at the fundamental frequency is obtained,

$$
\begin{aligned}
\Delta J_{C M G}^{\omega_{c}}= & \frac{1}{2}\left[\Delta \sigma_{\Delta \omega+\omega_{c}, k} \Delta \xi_{\Delta \omega, k}^{*}+\Delta \sigma_{\Delta \omega, k}^{*} \Delta \xi_{\Delta \omega+\omega_{c}, k}\right. \\
& \left.+\Delta \sigma_{\Delta \omega, k} \Delta \xi_{\Delta \omega-\omega_{c}, k}^{*}+\Delta \sigma_{\Delta \omega-\omega_{c}, k}^{*} \Delta \xi_{\Delta \omega, k}\right]+\Delta \sigma_{\omega_{c}, 0} \xi_{\text {ext }}
\end{aligned}
$$

The last term cancels when there is no externally applied electric field, and we get Eq. (11).

\section{APPENDIX C: EQUIVALENCE BETWEEN MCT AND CMC FOR SMALL CHOPPING FREQUENCIES}

In Appendix $\mathrm{A}$, we solve the general transport equations for a generation rate consisting of an arbitrary harmonic traveling wave on a much greater uniform background. The harmonic amplitudes of the photoconductivity and electric field can be rewritten as follows:

$$
\begin{aligned}
& \Delta \sigma_{\Omega, k}=g_{\Omega, k} \Delta \sigma_{\Omega, k}^{0}, \\
& \Delta \xi_{\Omega, k}=g_{\Omega, k} \Delta \xi_{\Omega, k}^{0},
\end{aligned}
$$

where $\Delta \sigma_{\Omega, k}^{0}$ and $\Delta \xi_{\Omega, k}^{0}$ do not depend on the harmonic amplitude of the generation rate, $g_{\Omega, k}$. Using Eqs. (C1) and (C2), the MGT current density given by Eq. (3) results in

$$
\Delta J_{M G T}=\frac{\Delta G_{0}^{2}}{2} \operatorname{Re}\left\{\Delta \sigma_{\Delta \omega, k}^{0}\left(\Delta \xi_{\Delta \omega, k}^{0}\right)^{*}\right\} .
$$

Similarly, the fundamental frequency component of the CMG current density, given by Eq. (11), becomes

$$
\Delta J_{C M G}^{\omega_{c}}=\frac{\Delta G_{0}^{2}}{4 \pi}\left[\begin{array}{c}
\Delta \sigma_{\Delta \omega+\omega_{c}, k}^{0}\left(\Delta \xi_{\Delta \omega, k}^{0}\right)^{*}+\left(\Delta \sigma_{\Delta \omega, k}^{0}\right)^{*} \Delta \xi_{\Delta \omega+\omega_{c}, k}^{0} \\
+\Delta \sigma_{\Delta \omega, k}^{0}\left(\Delta \xi_{\Delta \omega-\omega_{c}, k}^{0}\right)^{*}+\left(\Delta \sigma_{\Delta \omega-\omega_{c}, k}^{0}\right)^{*} \Delta \xi_{\Delta \omega, k}^{0}
\end{array}\right] .
$$

We assume a chopping frequency much smaller than the frequency difference between interfering beams $\left(\omega_{c} \ll \Delta \omega\right)$. Discarding $\omega_{c}$ in front of $\Delta \omega$ in the previous equation, we get

$$
\Delta J_{C M G}^{\omega_{c}}=\frac{\Delta G_{0}^{2}}{\pi} \operatorname{Re}\left\{\Delta \sigma_{\Delta \omega, k}^{0}\left(\Delta \xi_{\Delta \omega, k}^{0}\right)^{*}\right\} .
$$

Combining Eq. (C3) with Eq. (C5), we obtain the following equation:

$$
\Delta J_{M G T}=\frac{\pi}{2} \Delta J_{C M G}^{\omega_{c}}
$$

Taking the modulus on both sides of the previous identity, we finally get Eq. (6).

\section{REFERENCES}

${ }^{1}$ U. Haken, M. Hundhausen, and L. Ley, Appl. Phys. Lett. 63, 3066 (1993).

${ }^{2}$ U. Haken, M. Hundhausen, and L. Ley, J. Non-Cryst. Solids 164-166, 497 (1993).

${ }^{3}$ C. Witt, U. Haken, and M. Hundhausen, Jpn. J. Appl. Phys., Part 2 33, L1386 (1994).

${ }^{4}$ U. Haken, M. Hundhausen, and L. Ley, Phys. Rev. B 51, 10579 (1995)

${ }^{5}$ The dielectric relaxation time is given by the ratio of the steady-state photoconductivity and the material permittivity.

${ }^{6}$ R. A. Street, Hydrogenated Amorphous Silicon (Cambridge University Press, Cambridge, 1991).

${ }^{7}$ A. Merazga, S. Tobbeche, C. Main, A. Al-Shahrani, and S. Reynolds, J. Phys.: Condens. Matter 18, 3721 (2006).

${ }^{8}$ F. Ventosinos, N. Budini, C. Longeaud, and J. A. Schmidt, J. Phys. D: Appl. Phys. 44, 295103 (2011).

${ }^{9}$ F. Ventosinos, C. Longeaud, and J. A. Schmidt, J. Non-Cryst. Solids 358, 2031 (2012).

${ }^{10}$ C. Longeaud, F. Ventosinos, and J. A. Schmidt, J. Appl. Phys. 112, 023709 (2012).

${ }^{11}$ L. Kopprio, C. Longeaud, and J. Schmidt, J. Appl. Phys. 122, 085702 (2017).

${ }^{12}$ L. Kopprio, C. Longeaud, F. Ventosinos, and J. Schmidt, "Further insight into the oscillating photocarrier grating technique: Influence of the oscillation amplitude," Appl. Phys. B (submitted).

${ }^{13}$ V. Halpern, Philos. Mag. B 54, 473 (1986).

${ }^{14}$ J. Furlan, F. Smole, and P. Popović, MRS Proc. 297, 363 (1993).

${ }^{15}$ The precise shape of the CMG signal can be found by obtaining the higher order harmonics of the induced current density.

${ }^{16}$ W. Shockley and W. T. Read, Phys. Rev. 87, 835 (1952).

${ }^{17}$ J. G. Simmons and G. W. Taylor, Phys. Rev. B 4, 502 (1971). 